\title{
锰催化未活化烯烃的氢芳化反应
}

\author{
何恒驰 谢 劲* \\ (南京大学化学化工学院 配位化学国家重点实验室 江苏省先进有机材料重点实验室 南京 210023)
}

\section{Manganese-Catalyzed Hydroarylation of Unactivated Alkenes}

\author{
He, Hengchi Xie, Jin*
}

(State Key Laboratory of Coordination Chemistry, Jiangsu Key Laboratory of Advanced Organic Materials, School of Chemistry and Chemical Engineering, Nanjing University, Nanjing 210023)

过渡金属催化烯烃的官能团化已成为构建化学键 的高效策略. 由于未活化的烯烃反应活性低, 且对 $\mathrm{C}=$ C键的区域选择性控制通常较差, 因此与活化烯烃相比, 未活化烯烃的精确官能团化是该领域的一大挑战. 从反 应机制上看，原位形成的 $\mathrm{C}\left(\mathrm{sp}^{3}\right)-\mathrm{M}$ 中间体往往倾向于 进行 $\beta$-H 消除，并产生 Heck 型产物，这为选择性调控增 加了难度. 近年, 一种新的配位辅助策略被应用于过渡 金属催化的未活化烯烃氢芳化. 2010 年, Lautens 等 ${ }^{\left[{ }^{[} \text {实 }\right.}$ 现了 $\mathrm{Rh}$ 催化末端烯丙基砜/胺与芳基硼酸的氢芳化反 应, 通过 $\beta-\mathrm{H}$ 消除 $/ \mathrm{M}-\mathrm{H}$ 迁移再插入机制得到线性氢芳 化反应产物(Scheme 1a). 2018 年, Engle $\mathrm{e}^{[2]}$ 和南开大学赵 东兵课题组 ${ }^{[3-4]}$ 分别报道了 Pd 和 $\mathrm{Ni}$ 催化未活化烯烃的 氢芳化反应, 均需要利用双齿 $\mathrm{AQ}$ (8-氨基喹啉)导向基 稳定 $\mathrm{C}\left(\mathrm{sp}^{3}\right)-\mathrm{M}$ 中间体 (Scheme 1b). 因此, 发展新的催 化系统以实现含有不同官能团的未活化烯烃的精准的 氢芳化反应仍具有重大合成价值.

锰是一种地壳中含量丰富的过渡金属, 具有较好的 生物兼容性, 有望成为可持续化学领域中的新型过渡金 属催化剂. 2013 年以来, 中国科学院化学研究所王从洋 课题组 ${ }^{[5]}$ 开创性发展了芳烃的碳氢键活化策略, 原位生 成芳基锰中间体, 并成功应用于炔烃的氢芳化反应研 究. 此外, 对活化烯烃的锰催化氢芳化反应也于 2015 年 被报道 ${ }^{[6]}$. 然而芳基锰与未活化烯烃的反应活性极低, 仅有一例, 2018 年王从洋课题组 ${ }^{[7]}$ 实现的未活化烯烃的 氢芳化反应, 需要一个芳基酮来导向五元环锰的形成. 到目前为止, 还没有实现锰催化未活化烯烃与芳基硼酸 的氢芳化反应. 为此, 王从洋课题组 ${ }^{[8]}$ 近期报道了第一 (a) Rh-catalyzed hydroarylation of allyl sulfones/amines: previous work

$$
\begin{aligned}
& \mathrm{SO}_{2} \mathrm{R} / \mathrm{NR}^{1} \mathrm{R}^{2}+\operatorname{ArB}(\mathrm{OH})_{2} \quad \stackrel{\text { cat. }\left[\mathrm{Rh}(\mathrm{cod}) \mathrm{OH}_{2}\right.}{\mathrm{BINAP}} \\
& \mathrm{Ar} \sim \mathrm{SO}_{2} \mathrm{R} / \mathrm{NR}^{1} \mathrm{R}^{2}
\end{aligned}
$$

(b) Pd-/Ni-catalyzed AQ-assisted hydroarylation: previous work

$$
\text { base }
$$

(c) Mn-catalyzed hydroarylation of diverse alkenes: this work

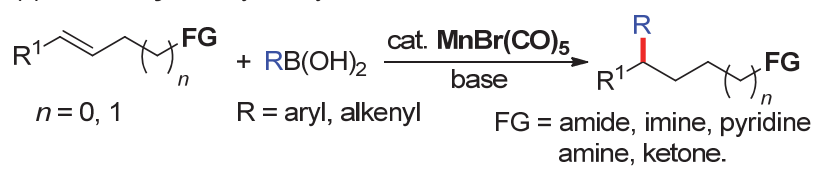

图式 1 过渡金属催化未活化烯烃与嗍酸的氢芳化反应 Scheme 1 Transition-metal-catalyzed hydroarylation of unactivated alkenes with boronic acids

例锰催化的未活化烯烃与芳基硼酸的高选择性氢芳化 反应. 该反应适用于酰胺、亚胺、吡啶、氨基、羰基等 官能团, 且相较于以往采用的铑、钯、镍催化剂有着极 好的 $\gamma-/ \delta$-区域选择性.

王从洋课题组以内烯烃 1a 和苯基硼酸为模板反应, 以 95\%产率制得了区域选择性极好的 $\gamma$-芳基取代产物 3aa (Scheme 2). 对照实验表明, 没有锰催化的情况下 没有产物，而没有碱的情况下产率将降低至 $23 \%$. 碳酸 钾作碱可以得到最优产率, 底物比例对产率没有明显影

* Corresponding author. E-mail: xie@nju.edu.cn. Published online October 4, 2020. 


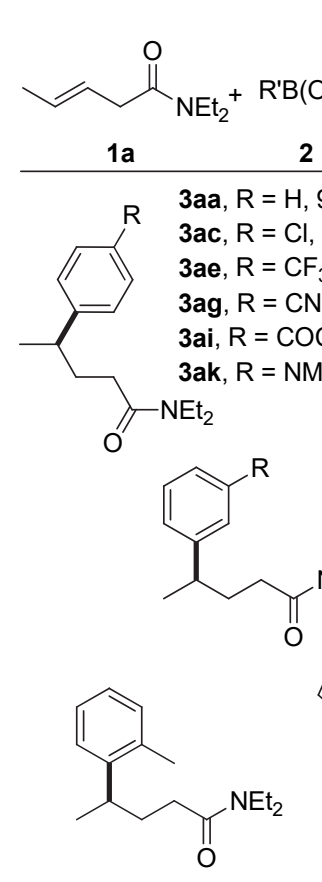

3ap, $89 \%(97 / 3)$
$\operatorname{MnBr}(\mathrm{CO})_{5}(10 \mathrm{~mol} \%)$

)$_{2} \underset{\mathrm{THF}, 100^{\circ} \mathrm{C}, 12 \mathrm{~h}}{\stackrel{\mathrm{K}_{2} \mathrm{CO}_{3}(1.0 \text { equiv. })}{\mathrm{R}^{\prime}}}$

$\%(97 / 3) ; 3 a b, R=F, 91 \%(98 / 2)$

2)

)

$94 \%$ (98/2); 3af, $\mathrm{R}=\mathrm{CO}_{2} \mathrm{Me}, 92 \%(99 / 1)$

$98 \%(99 / 1) ; 3 a h, \mathrm{R}=\mathrm{NO}_{2}, 91 \%(99 / 1)$

$\mathrm{H}_{3}, 99 \%(93 / 7) ; 3 a j, \mathrm{R}=\mathrm{CHO}, 80 \%(97 / 3)$
(9)

3am, $\mathrm{R}=\mathrm{OMe}, 93 \%(99 / 1)$
3an, $\mathrm{R}=\mathrm{Me}, 91 \%(99 / 1)$

$\mathrm{NEt}_{2}$ 3ao, $\mathrm{R}=\mathrm{NO}_{2}, 91 \%(99 / 1)$<smiles>CCN(CC)C(=O)CCC(C)c1ccc2[nH]ccc2c1</smiles><smiles>CCNC(=O)CCC(C)/C=C/c1ccccc1</smiles>

3ar, $97 \%(99 / 1)$<smiles>[R]C(CC)C(=O)NCC</smiles>

图式 2 酰胺导向内烯烃的 $\gamma$-氢芳化反应中嗍酸适用范围

Scheme 2 Scope with respect to boronic acids in the $\gamma$-selective hydroarylation of alkenes with amide groups

响. 一系列带有卤素、三氟甲基、酯基、氰基、硝基、 酮基、醛基、氨基、羟基、酰胺基等官能团的芳基硼酸 都可以顺利生成目标产物, 为进一步修饰提供了便利. 不仅如此, 邻位和间位取代的芳基嗍酸以及菜基和杂环 嗍酸都可以顺利进行氢芳化. 值得一提的是, 烯基硼酸 也适用并能给出极好的区域和 $E / Z$ 选择性. 对烯烃底物 的进一步研究发现, 该反应对一系列底物均有较好的适 用性, 如酰胺上的 $N$-取代和未取代的不同底物, 以及内 烯烃、末端烯烃或 1,1-二取代烯烃都能顺利进行反应 (Scheme 3).

为了进一步扩大该反应的应用范围, 王从洋课题组 还尝试了在相同条件下以 $\gamma, \delta$-不饱和亚胺为底物进行反 应(Scheme 4), 结果表明芳基上不同取代基均能得到很 好的产率, 并在水解后得到 $\delta$-取代酮. 缺电子芳基硼酸 需要更高的反应温度. 此外, 使用间位取代的芳基硼酸 也有很好的产率.

使用高烯丙基吡啶可以得到专一的 $\delta$-取代产物, 而 有一个未保护的亚氨基的高烯丙基亚胺也可以顺利生 成高区域选择性的产物, 这一点与使用 $\mathrm{Pd}, \mathrm{Ni}, \mathrm{Rh}$ 催化 剂时不同.

作者提出了可能的反应机理(Scheme 5): 首先由
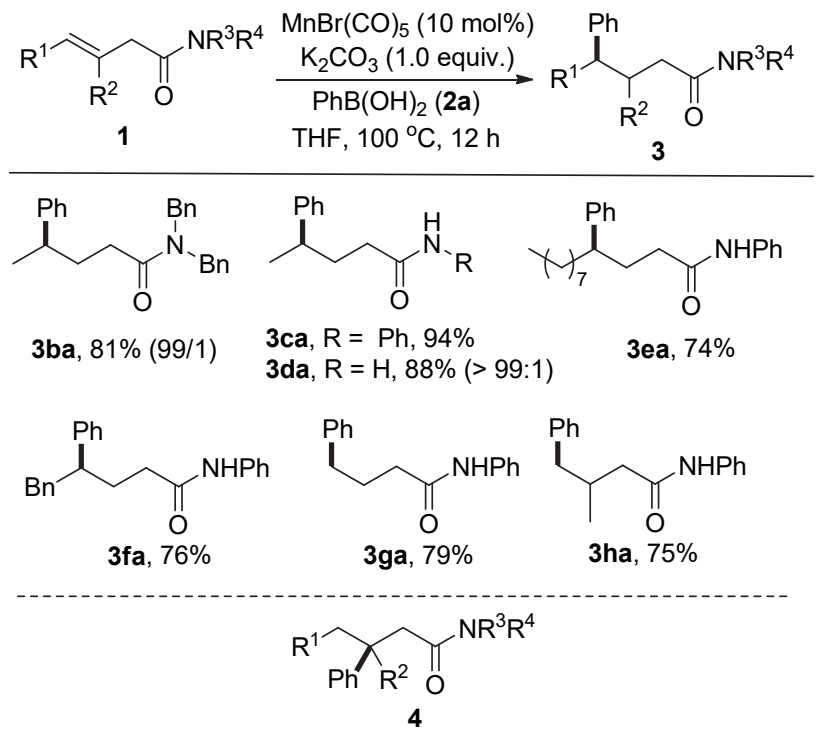

图式 3 酰胺基烯烃的 $\gamma$-氢芳化反应中烯烃取代基适用范围 Scheme 3 Scope with respect to alkenes in the $\gamma$-selective hydroarylation of alkenes with amide groups

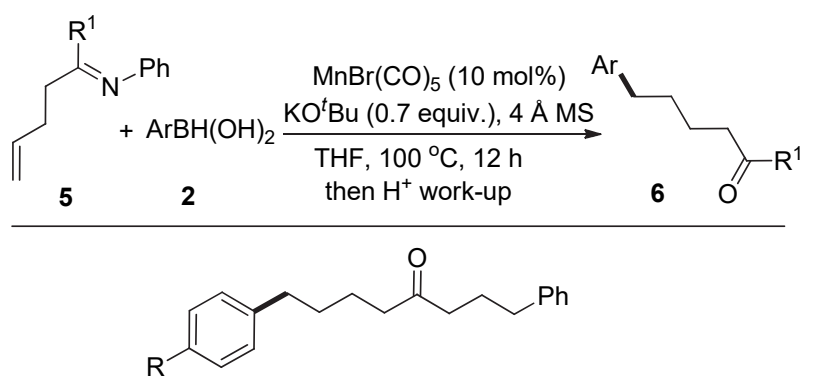

6aa, $\mathrm{R}=\mathrm{H}, 79 \%$; 6ab, $\mathrm{R}=\mathrm{Me}, 80 \%$; $\mathbf{6 a c}, \mathrm{R}=\mathrm{Ph}, 80 \%$;

6ad, $\mathrm{R}=\mathrm{F}, 63 \%$; 6ae, $\mathrm{R}=\mathrm{Cl}, 79 \%$; 6 af, $\mathrm{R}=\mathrm{Br}, 64 \%$;

6ag, $\mathrm{CF}_{3}, 73 \%$; 6 ah, $\mathrm{CO}_{2} \mathrm{Me}, 45 \%$; 6 ai, $\mathrm{R}=\mathrm{CN}, 40 \%$

图式 4 酰胺基烯烃的 $\delta$-氢芳化反应适用的底物范围 Scheme 4 Scope of the $\delta$-selective hydroarylation of alkenes with imine groups

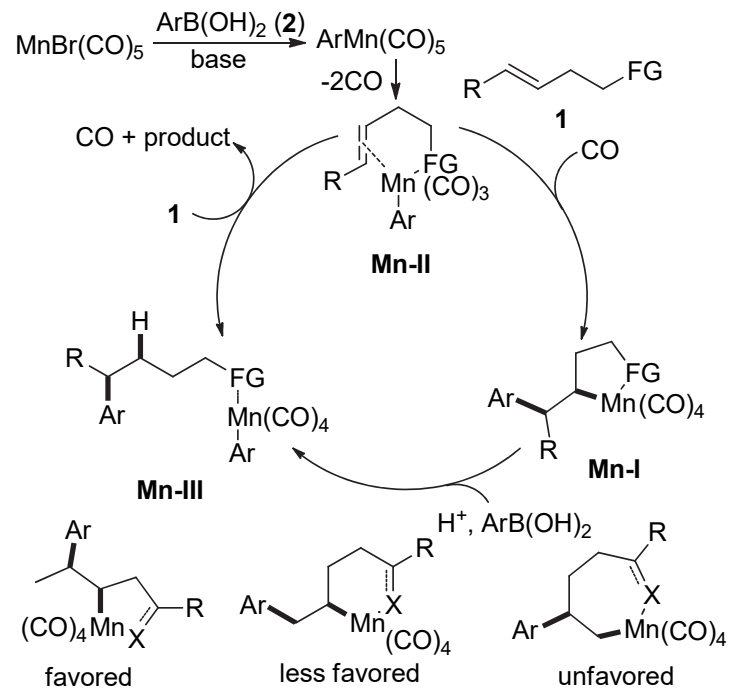

图式 5 推测的反应机理

Scheme 5 A proposed reaction mechanism 
$\operatorname{MnBr}(\mathrm{CO})_{5}$ 和芳基硼酸 $\mathbf{2}^{[9-10]}$ 在碱的辅助下通过转金属 化生成 $\operatorname{ArMn}(\mathrm{CO})_{5}$, 接着失去 $\mathrm{CO}$ 配体, 与烯烃 1 配位 形成 Mn-II. 此时烯烃发生迁移插入反应生成 Mn-I, 这 一步形成环的大小决定了区域选择性. 接下来质子化和 进一步的转金属化生成 Mn-III, 该中间体与 1 进行配体 交换得到产物.

总之, 王从洋课题组发展了第一例锰催化未活化烯 烃和芳基喼酸的高选择性氢芳化反应, 无需引入双齿 $\mathrm{AQ}$ 导向基便可实现区域可控. 该反应用锰作为催化剂, 原料简单易得, 具有广泛的官能团耐受性和优良的区域 选择性控制等优点, 展现出锰的独特性与优越性, 为不 饱和烯烃的官能团修饰提供了有效方法.

\section{References}

[1] Tsui, G. C.; Lautens, M. Angew. Chem., Int. Ed. 2010, 49, 8938.

[2] Matsuura, R.; Jankins, T. C.; Hill, D. E.; Yang, K. S.; Gallego, G. M.; Yang, S.; He, M.; Wang, F.; Marsters, R. P.; McAlpine, I.; Engle, K. M. Chem. Sci. 2018, 9, 8363.

[3] Lv, H.; Xiao, L. J.; Zhao, D.; Zhou, Q.-L. Chem. Sci. 2018, 9 , 6839.

[4] Lv, H.; Kang, H.; Zhou, B.; Xue, X.; Engle, K. M.; Zhao, D. Nat. Commun. 2019, 10, 5025.

[5] Zhou, B.; Chen, H.; Wang, C. J. Am. Chem. Soc. 2013, 135, 1264.

[6] Liu, W.; Zell, D.; John, M.; Ackermann, L. Angew. Chem., Int. Ed. 2015, 54, 4092.

[7] Hu, Y.; Zhou, B.; Chen, H.; Wang, C. Angew. Chem., Int. Ed. 2018, $57,12071$.

[8] Liu, T.; Yang, Y.; Wang, C. Angew. Chem., Int. Ed. 2020, 59, 14256.

[9] Yan, Z.; Yuan, X. A.; Zhao, Y.; Zhu, C.; Xie, J. Angew. Chem., Int. Ed. 2018, 57, 12906.

[10] Wang, D.; Dong, J.; Fan, W.; Yuan, X.-A.; Han, J.; Xie, J. Angew. Chem., Int. Ed. 2020, 59, 8430. 